\title{
Phase transition in nanomagnetite
}

\author{
I. Dézsi, ${ }^{1, a)}$ Cs. Fetzer, ${ }^{1}$ Á. Gombkötő, ${ }^{1}$ I. Szücs, ${ }^{1}$ J. Gubicza, ${ }^{2}$ and T. Ungár ${ }^{2}$ \\ ${ }^{1}$ KFKI Research Institute for Particle and Nuclear Physics, P.O. Box 49, H-1525 Budapest 114, Hungary \\ ${ }^{2}$ Department of Materials Physics, ELTE, Institute of Physics, P.O. Box 32, H-1518 Budapest, \\ Hungary
}

(Received 19 October 2007; accepted 30 March 2008; published online 29 May 2008)

\begin{abstract}
Recently, the application of nanosized magnetite particles became an area of growing interest for their potential practical applications. Nanosized magnetite samples of 36 and $9 \mathrm{~nm}$ sizes were synthesized. Special care was taken on the right stoichiometry of the magnetite particles. Mössbauer spectroscopy measurements were made in $4.2-300 \mathrm{~K}$ temperature range. The temperature dependence of the intensities of the spectral components indicated size dependent transition taking place in a broad temperature range. For nanosized samples, the hyperfine interaction values and their relative intensities changed above the Verwey transition temperature value of bulk megnetite. The continuous transition indicated the formation of dendritelike granular assemblies formed during the preparation of the samples. (C) 2008 American Institute of Physics. [DOI: 10.1063/1.2937252]
\end{abstract}

\section{INTRODUCTION}

Magnetite, $\mathrm{Fe}_{3} \mathrm{O}_{4}$, is a prototype of inverse spinels. At room temperature, it has a cubic spinel structure with a lattice parameter of $8.397 \AA$. The unit cell consists of a cage of $32 \mathrm{O}^{2+}$ ions arranged in a face centered cubic lattice. Each unit cell has eight $\mathrm{Fe}^{3+}$ ions at the tetrahedral site (A sites) with four nearest neighbor $\mathrm{O}^{2+}$ ions, and eight $\mathrm{Fe}^{3+}$ and eight $\mathrm{Fe}^{2+}$ ions at the octahedral sites (B sites) with six nearest neighbor $\mathrm{O}^{2+}$ ions. Magnetite is a relatively good conductor at room temperature. On cooling below $120 \mathrm{~K}$, its conductivity sharply drops by two orders of magnitude. Verwey evidenced $^{1}$ this sharp anomaly in 1939. The transition was explained ${ }^{2}$ by a change from a dynamic disorder of electrons hopping on $\mathrm{B}$ sites above the Verwey transition $\left(T_{V}\right)$ to a long range order of the $\mathrm{B}$ site cations in alternate plans of $\mathrm{Fe}^{3+}$ and $\mathrm{Fe}^{2+}$ ions. On cooling below $T_{V}$, the change from cubic (space group $F d \overline{3} m$ ) to monoclinic (space group $P 2 / c$ ) structure yields a change in the local symmetry around the cations. ${ }^{3}$ The Verwey-Mott model assumes a purely electronic mechanism for the transition. ${ }^{4}$ Nevertheless, despite extensive studies, the mechanism responsible for the transition is still not completely understood..$^{5}$

Recently, the application of small particle or nanoparticle magnetite became an area of growing interest due to their potential practical applications. In the case of magnetic nanostructures, data storage is a potential application. ${ }^{6}$ Also, the application of magnetic nanostructures in magnetic resonance imaging and magnetically steered drug delivery ${ }^{7-9}$ is expected.

Mössbauer spectrometry is a very sensitive method that provides information on the Fe ions that are located at different lattice positions, which results in different hyperfine parameters. ${ }^{10}$ Below $T_{V}$, the spectrum changed significantly. ${ }^{11,12}$ In the case of nanomagnetite, the dependence of the transition on the particle size is interesting but contradicting. A decrease ${ }^{13,14}$ and an increase ${ }^{15}$ in $T_{V}$ were

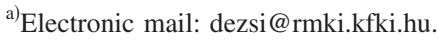

measured depending on the size of the samples. However, the studies were performed only at some temperature values and not in a broad temperature range, and the preparation of the samples studied were made by using different methods. No transition was observed below $50 \mathrm{~nm}$ size. ${ }^{14}$ A change in the slope of the zero-field-cooled magnetization graph for 10 and $24 \mathrm{~nm}$ sized samples at $100 \mathrm{~K}$ was attributed to Verwey transition. ${ }^{16}$ Recently, the fractal formation (dendriticlike self-assembly) by magnetite nanoparticles was observed on porous silicon in aqueous media. ${ }^{17}$

Because of the different results obtained using special preparation techniques and with the samples measured at some temperature values, we intend to perform measurement on nanomagnetite samples synthesized by using classical methods. Our aim was to prepare bulk and nanomagnetite samples and measure the temperature dependence of Mössbauer spectra to compare the results and conclude on the temperature dependence of the crystal transformation for 9 and $36 \mathrm{~nm}$ sized magnetite samples. Up until now, the Mössbauer spectra of nanomagnetite samples have not been measured systematically in a broad temperature range. We measured the samples in a broad temperature range between 4.2 and $300 \mathrm{~K}$.

\section{EXPERIMENTAL DETAILS}

Bulk magnetite crystals were prepared by reducing melted hematite in $\mathrm{CO} / \mathrm{CO}_{2}$ atmosphere. ${ }^{18}$ The reduction and cooling down took place for several hours. The nanomagnetite samples had to be synthesized by different chemical methods depending on the particle size. The sample of $36 \mathrm{~nm}$ thickness was prepared by the method described in Ref. 19. The sample of $9 \mathrm{~nm}$ size was prepared by the precipitation of magnetite from the basic aqueous solution of $\mathrm{FeCl}_{2}$ and $\mathrm{FeCl}_{3}$, as was described earlier. ${ }^{20}$ After the syntheses, the iron content of the segregated samples was chemically analyzed. The results proved the stoichiometric $\mathrm{Fe}_{3} \mathrm{O}_{4}$ compositions. The surface of the samples was covered with oleic acid and oleylamine to keep nanodispersion and to 
avoid oxidation on the surface. The structure of the nanomagnetite samples was determined by the x-ray diffraction method. The diffraction results supported the cubic structure. The size distribution was determined by $\mathrm{x}$-ray line profile analysis. The full width at half maximum (FWHM) of the profiles was plotted as a function of the length of the diffraction vector (Williamson-Hall method). These plots showed that the FWHM values are independent of the indices of reflections, which indicates that there are no significant lattice strains in the structure. In this case, the broadening of the diffraction profiles is caused by the smallness of the crystallites (grains) and the crystallite size distribution can be evaluated from a single profile. The crystallite size distribution was determined from the profile of (400) reflection because this peak was relatively strong and did not overlap with neighboring lines. The peak profile was evaluated by the convolutional multiple whole profile fitting method, which is described in detail elsewhere. ${ }^{21}$ In this procedure, the measured line profile was fitted by the convolution of the instrumental peak and the physical profile resulted by the crystallite size distribution. The instrumental profile was measured on a LaB6 peak profile standard material. In the fitting procedure the size distribution density $[f(x)]$ was assumed to be a lognormal function, which is given by

$$
f(x)=\frac{1}{\sqrt{2 \pi} \sigma} \frac{1}{x} \exp \left[-\frac{[\ln (x / m)]^{2}}{2 \sigma^{2}}\right],
$$

where $x$ is the crystallite size, and $m$ and $\sigma$ are the median and variance of the size distribution, respectively. The variance characterizes the width of the size distribution. The line profile fitting gives the values of $m$ and $\sigma$ directly. From $m$ and $\sigma$, the mean crystallite size can be determined as ${ }^{22}$

$$
\langle x\rangle=m \exp \left(\sigma^{2} / 2\right) .
$$

X-ray line profile analysis gives $m=9 \pm 1 \mathrm{~nm}, \quad \sigma$ $=0.31 \pm 0.04$, and $\langle x\rangle=9 \pm 1 \mathrm{~nm}$ for the $9 \mathrm{~nm}$ sample, and $m=34 \pm 4 \mathrm{~nm}, \sigma=0.35 \pm 0.04$, and $\langle x\rangle=36 \pm 4 \mathrm{~nm}$ for the $36 \mathrm{~nm}$ sample. It can be seen that the width of the size distribution is the same within the experimental error, while the mean size value is four times higher for the larger sample. It was shown for different nanosized materials that the bar diagram of the crystallite size distribution obtained from transmission electron microscopy micrographs and the size distribution density function, $f(x)$, determined by x-ray analysis give the same results. ${ }^{22}$

The Mössbauer absorber samples were prepared in a very pure $\mathrm{Ar}$ atmosphere. To keep the dispersion of the nanoparticles, they were mixed with fine BN powder. The measurements at low temperatures were made in a cryostat containing liquid $\mathrm{N}_{2}$ or He. The application of the Mössbauer spectrometry to determine the phase transition is very effective, because the method is an order-sensitive technique due to the strong dependence of the hyperfine parameters on the Fe-atom surroundings. Therefore, the local positions, the interaction of ions, and their oxidation state can simultaneously be determined. The Mössbauer spectra were measured using the spectrometer in a constant velocity mode. A single line source of $50 \mathrm{mCi}{ }^{57} \mathrm{Co}$ in a Rh matrix was used. The spectra

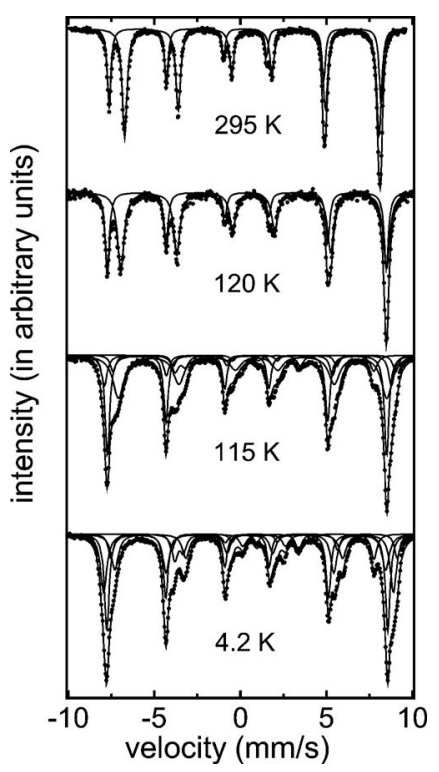

FIG. 1. Mössbauer spectra of bulk magnetite.

were fitted by using a least-squares fitting program. The isomer shift $\delta$ values in $\mathrm{mm} / \mathrm{s}$ are given relative to that of $\alpha$-iron at room temperature.

\section{RESULTS AND DISCUSSION}

The Mössbauer spectra of the bulk magnetite sample were measured at different temperatures, and four of them are shown in Fig. 1. The spectra were measured to perform a reliable comparison of the hyperfine parameters of bulk and nanomagnetite samples. The spectra above $T_{V}$ consist of two components corresponding to $\mathrm{Fe}^{3+}$ and the intermediate ions in the lattice denoted by the average valence state $\mathrm{Fe}^{2.5+}$. The intensity ratio of $\mathrm{Fe}^{3+} / \mathrm{Fe}^{2.5+}$ was $1: 2$ at room temperature, as can be expected for a stoichiometric sample assuming the same Lamb Mössbauer factor of the components. Below $T_{V}$, the spectral shape changed considerably, as was already observed earlier. ${ }^{12,23}$ Below $T_{V}$, the fit was made earlier by four components. ${ }^{24}$ However, in most cases, the spectra were fitted by five components, ${ }^{12,25}$ resulting in the best result. Nevertheless, even more, six and nine components were supposed, ${ }^{26,27}$ but the exact reason for the existence of these components was not given. We fitted our spectra with five components below $T_{V}$. The parameters obtained are compiled in Table I. Below $T_{V}$, component a and component $\mathrm{b}_{1}$ can be attributed to $\mathrm{Fe}^{3+}$ in tetrahedral and octahedral positions, respectively, and $\mathrm{b}_{2}, \mathrm{~b}_{3}$, and $\mathrm{b}_{4}$ to $\mathrm{Fe}^{2+}$ in octahedral position. This attribution is in agreement with those published. ${ }^{12,25}$ The appearance of the five components, some with different electric quadrupole splitting values, can be attributed to a complex monoclinic symmetry resulting in an electric field gradient at the cation sites in different sublattices. According to the refined model, the monoclinic cell has an orthorombic Pmca pseudosymmetry, constraining the atomic positions. Therefore, the lattice contains four iron atoms in subcells of the differently distorted octahedrons. ${ }^{28} \mathrm{By}$ fitting the spectra with five components below $T_{V}$, the hyperfine interaction parameter values measured by us are close to 
TABLE I. Mössbauer parameters of bulk magnetite measured at different temperatures: isomer shift $(\delta)$, quadrupole splitting $(\mathrm{QS})$ given in $\mathrm{mm} / \mathrm{s}$, internal magnetic field $H$ given in T, and relative intensity (RI) values of the spectrum components given as percentages. Ions in (a) tetraheral and (b) octahedral positions.

\begin{tabular}{cccccc}
\hline \hline \multirow{2}{*}{ Temperature } & Component & $\delta$ & QS & $H$ & RI \\
\hline \multirow{2}{*}{$295 \mathrm{~K}$} & $\mathrm{a}$ & $0.27(3)$ & $0.00(3)$ & $49.0(1)$ & $34(1)$ \\
& $\mathrm{b}$ & $0.66(3)$ & $0.00(3)$ & $45.9(1)$ & $66(1)$ \\
\multirow{2}{*}{$120 \mathrm{~K}$} & & & & & \\
& $\mathrm{a}$ & $0.37(3)$ & $0.00(3)$ & $50.3(1)$ & $36(1)$ \\
& $\mathrm{b}$ & $0.77(3)$ & $0.00(3)$ & $47.9(1)$ & $64(2)$ \\
$115 \mathrm{~K}$ & & & & & \\
& $\mathrm{a}$ & $0.38(3)$ & $0.01(3)$ & $50.4(1)$ & $37(1)$ \\
& $\mathrm{b} 1$ & $0.47(3)$ & $-0.10(3)$ & $51.6(1)$ & $11(1)$ \\
& $\mathrm{b} 2$ & $0.77(3)$ & $-0.02(3)$ & $50.5(1)$ & $16(1)$ \\
& $\mathrm{b} 3$ & $0.96(3)$ & $-0.3(3)$ & $48.2(1)$ & $27(1)$ \\
& $\mathrm{b} 4$ & $0.98(3)$ & $1.88(3)$ & $35.7(1)$ & $9(1)$ \\
& & & & & \\
& $\mathrm{a}$ & $0.39(3)$ & $0.01(3)$ & $50.7(1)$ & $33(1)$ \\
& $\mathrm{b} 1$ & $0.51(3)$ & $-0.08(3)$ & $52.3(1)$ & $27(1)$ \\
& $\mathrm{b} 2$ & $0.81(3)$ & $-0.04(3)$ & $51.6(1)$ & $9(1)$ \\
& $\mathrm{b} 3$ & $0.98(3)$ & $-0.81(3)$ & $48.8(1)$ & $19(2)$ \\
& $\mathrm{b} 4$ & $1.02(3)$ & $1.94(3)$ & $35.9(1)$ & $11(1)$ \\
\hline \hline
\end{tabular}

those obtained earlier for bulk magnetite. According to Ref. 23, two different $\mathrm{Fe}^{3+}$ and four $\mathrm{Fe}^{2+}$ lattice positions exist in the lattice. Therefore, six Mössbauer spectral positions are expected to appear. However, only five components can be distinguished. The reason could be that two $\mathrm{Fe}^{2+}$ components have very close hyperfine parameters and they could not be resolved.

Four characteristic Mössbauer spectra of the $36 \mathrm{~nm}$ thick sample are shown in Fig. 2. The spectrum measured at room temperature is very similar to the spectrum of the bulk phase. The only difference is that the linewidths are slightly broader. The shape of the spectra gradually changes at lower temperatures and the $\mathrm{Fe}^{3+} / \mathrm{Fe}^{2.5+}$ intensity ratio begins to increase. The spectra from $150 \mathrm{~K}$ can be fitted by six compo-

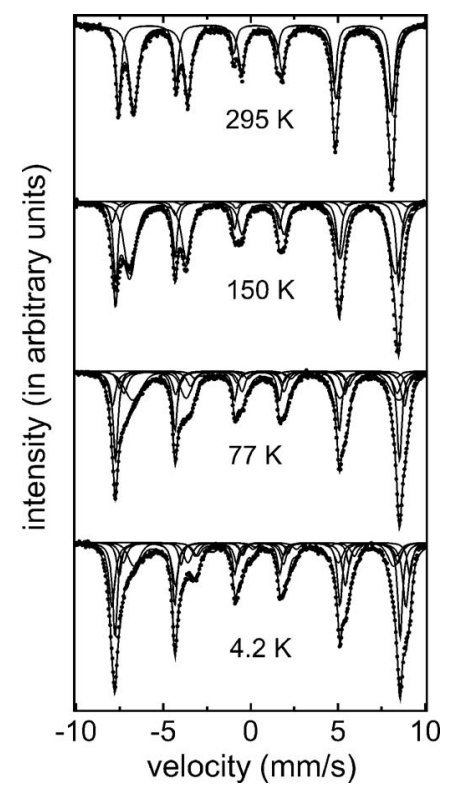

FIG. 2. Mössbauer spectra of $36 \mathrm{~nm}$ sized magnetite.
TABLE II. Mössbauer parameters of $36 \mathrm{~nm}$ magnetite measured at different temperatures: isomer shift $(\delta)$, QS given in $\mathrm{mm} / \mathrm{s}, H$ given in T, and RI values of the spectrum components given as percentages.

\begin{tabular}{|c|c|c|c|c|c|}
\hline Temperature & Component & $\delta$ & QS & $H$ & RI \\
\hline \multirow[t]{2}{*}{$295 \mathrm{~K}$} & a & $0.27(3)$ & $0.00(3)$ & $48.7(1)$ & $33(1)$ \\
\hline & b & $0.65(3)$ & $0.00(3)$ & $45.6(1)$ & $67(1)$ \\
\hline \multirow[t]{6}{*}{$140 \mathrm{~K}$} & a & $0.34(3)$ & $0.01(3)$ & $50.2(1)$ & $30(1)$ \\
\hline & b1 & $0.45(3)$ & $-0.02(3)$ & $51.5(1)$ & $10(1)$ \\
\hline & b2 & $0.50(3)$ & $-0.01(3)$ & $50.7(1)$ & $3(1)$ \\
\hline & b3 & $0.90(3)$ & $-0.58(3)$ & $48.3(1)$ & $6(1)$ \\
\hline & b4 & $0.95(3)$ & $1.85(3)$ & $35.7(1)$ & $0(1)$ \\
\hline & b5 & $0.74(3)$ & $0.10(3)$ & $46.7(1)$ & $51(1)$ \\
\hline \multirow[t]{6}{*}{$77 \mathrm{~K}$} & a & $0.39(3)$ & $0.02(3)$ & $50.5(1)$ & $32(1)$ \\
\hline & b1 & $0.49(3)$ & $-0.05(3)$ & $51.9(1)$ & $22(1)$ \\
\hline & b2 & $0.60(3)$ & $-0.02(3)$ & $51.2(1)$ & $7(1)$ \\
\hline & b3 & $0.94(3)$ & $-0.75(3)$ & $48.6(1)$ & $13(1)$ \\
\hline & b4 & $1.00(3)$ & $1.85(3)$ & $35.8(1)$ & $3(1)$ \\
\hline & b5 & $0.84(3)$ & $0.16(3)$ & 47.1(1) & $23(2)$ \\
\hline \multirow[t]{6}{*}{$4.2 \mathrm{~K}$} & $\mathrm{a}$ & $0.42(3)$ & $0.01(3)$ & $50.7(1)$ & $30(2)$ \\
\hline & b1 & $0.50(3)$ & $0.08(3)$ & $52.3(1)$ & $27(1)$ \\
\hline & b2 & $0.68(3)$ & $-0.04(3)$ & $51.6(1)$ & $11(1)$ \\
\hline & b3 & $0.97(3)$ & $-0.83(3)$ & $48.8(1)$ & $10(1)$ \\
\hline & b4 & $1.02(3)$ & $1.90(3)$ & $35.9(1)$ & $6(1)$ \\
\hline & b5 & $0.85(3)$ & $0.05(3)$ & $47.4(1)$ & $16(1)$ \\
\hline
\end{tabular}

nents. The fit values are included in Table II. The hyperfine parameters of the $a$ and $b_{1}$ components are very close to the values of bulk magnetite measured below $T_{V}$. The parameter values of $b_{3}, b_{4}$, and $b_{5}$ are also close to those obtained for the bulk sample. Hovewer, it was necessary to introduce a sixth sextet component $b_{2}$ to get the proper fit. This component was not purely Lorentzian, but it had a Voigt shape and the parameter values are close to those measured for $\mathrm{Fe}^{3+}$. The change in the hyperfine parameters and relative intensities took place surprisingly in a broad temperature range, not as they were observed for the bulk sample. The maximum $\mathrm{Fe}^{3+} / \mathrm{Fe}^{2+}$ values measured at $4.2 \mathrm{~K}$ indicate no oxidation effect. The fitted values are deployed in Table II. The $\mathrm{Fe}^{3+} /\left(\mathrm{Fe}^{2+}+\mathrm{Fe}^{3+}\right)$ ratios are shown in Fig. 4. The Mössbauer spectra of the $9 \mathrm{~nm}$ thick sample are shown in Fig. 3. The fitted values are listed in Table III. It is clearly seen that the intensity of the $\mathrm{Fe}^{2.5+}$ component decreases already at $210 \mathrm{~K}$. The hyperfine split values taking into account the different temperature values of the measurements are near those measured in the other samples. The $\mathrm{Fe}^{3+} /\left(\mathrm{Fe}^{2+}+\mathrm{Fe}^{3+}\right)$ intensity ratios are shown in Fig. 4. The phase transition begins at a higher temperature than in the bulk and the $36 \mathrm{~nm}$ sized sample. In addition, the transition to the monoclinic phase is complete at lower temperature as in bulk crystals. In the case of the $9 \mathrm{~nm}$ sized sample, the transition is more intensive at higher temperature values, as shown in Fig. 4.

It is known that in nonstoichiometric magnetite samples (e.g., containing $\gamma-\mathrm{Fe}_{2} \mathrm{O}_{3}$ in various concentration), $T_{V}$ is reduced from $120 \mathrm{~K}$ and a decrease in resistivity with increasing nonstoichiometry was observed. ${ }^{29} \mathrm{For}^{\mathrm{F} e} \mathrm{H}_{-\delta} \mathrm{O}_{4}$, the critical value $\delta=0.0039$ was determined. ${ }^{30}$ Above this value, $T_{V}$ shifts downward. The measured intensity ratios of the spec- 


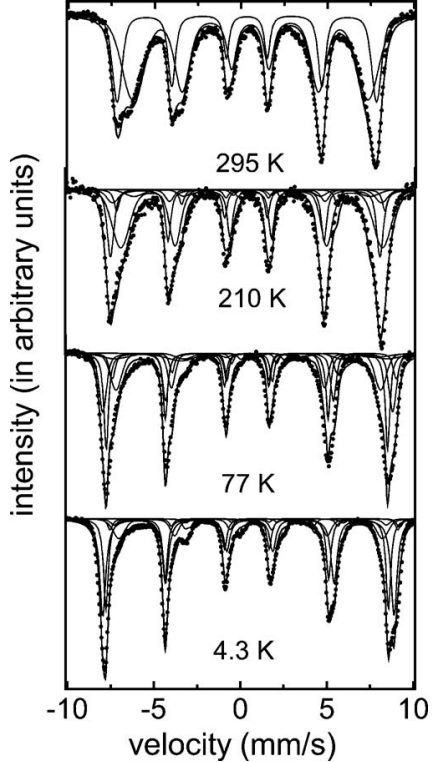

FIG. 3. Mössbauer spectra of $9 \mathrm{~nm}$ sized magnetite.

tral components in our samples exclude nonstoichiometry above this $\delta$ value. Recently, a mechanism was proposed for the formation of charge ordered surface states, providing an explanation for the increase in the phase transition temperature at the magnetic surface. ${ }^{31}$ Earlier, Anderson ${ }^{32}$ calculated much higher Verwey temperatures, taking into account the long and short range charge ordering in magnetite. For our 9 and $36 \mathrm{~nm}$ sized samples, the crystal structure is cubic at room temperature and the components of the Mössbauer spectra have the same isomer shift values as the sextet components of the bulk sample. This indicates that the samples

TABLE III. Mössbauer parameters of $9 \mathrm{~nm}$ magnetite measured at different temperatures: isomer shift $(\delta)$, QS given in $\mathrm{mm} / \mathrm{s}, H$ given in T, and RI values of the spectrum components given as percentages.

\begin{tabular}{|c|c|c|c|c|c|}
\hline Temperature & Component & $\delta$ & QS & $H$ & RI \\
\hline \multirow[t]{2}{*}{$295 \mathrm{~K}$} & $\mathrm{a}$ & $0.29(3)$ & $0.00(3)$ & 47.1(1) & $33(1)$ \\
\hline & $\mathrm{b}$ & $0.61(3)$ & $0.00(3)$ & 44.6(1) & $67(1)$ \\
\hline \multirow[t]{6}{*}{$210 \mathrm{~K}$} & $\mathrm{a}$ & $0.32(3)$ & $-0.03(3)$ & $48.3(1)$ & $23(1)$ \\
\hline & b1 & $0.41(3)$ & $-0.01(3)$ & $48.2(1)$ & $11(1)$ \\
\hline & b2 & $0.52(3)$ & $-0.08(3)$ & $42.7(1)$ & $6(1)$ \\
\hline & b3 & $0.87(3)$ & $-0.49(3)$ & 48.1(1) & $6(1)$ \\
\hline & b4 & $0.92(3)$ & $1.83(3)$ & $35.5(1)$ & $0(1)$ \\
\hline & b5 & $0.67(3)$ & $0.06(3)$ & $46.3(1)$ & $54(2)$ \\
\hline \multirow[t]{6}{*}{$77 \mathrm{~K}$} & $\mathrm{a}$ & $0.38(3)$ & $0.01(3)$ & $50.3(2)$ & $33(1)$ \\
\hline & b1 & $0.47(3)$ & $-0.03(3)$ & $51.8(1)$ & $23(1)$ \\
\hline & b2 & $0.62(3)$ & $-0.02(3)$ & $51.1(1)$ & $9(1)$ \\
\hline & b3 & $0.95(3)$ & $-0.70(3)$ & $48.5(1)$ & $12(1)$ \\
\hline & b4 & $1.02(3)$ & $1.87(3)$ & $35.6(1)$ & 2(1) \\
\hline & b5 & $0.85(3)$ & $0.13(3)$ & $47.0(1)$ & $21(1)$ \\
\hline \multirow[t]{6}{*}{$4.2 \mathrm{~K}$} & $\mathrm{a}$ & $0.41(3)$ & $-0.01(3)$ & $50.5(1)$ & $29(1)$ \\
\hline & b1 & $0.49(3)$ & $0.09(3)$ & $52.1(1)$ & $26(2)$ \\
\hline & b2 & $0.68(3)$ & $0.01(3)$ & $51.5(1)$ & $12(2)$ \\
\hline & b3 & $0.96(3)$ & $-0.90(3)$ & $48.6(2)$ & $10(1)$ \\
\hline & b4 & $1.00(3)$ & $1.95(3)$ & $35.7(1)$ & $5(1)$ \\
\hline & b5 & $0.86(3)$ & $0.01(3)$ & $47.3(1)$ & $18(1)$ \\
\hline
\end{tabular}

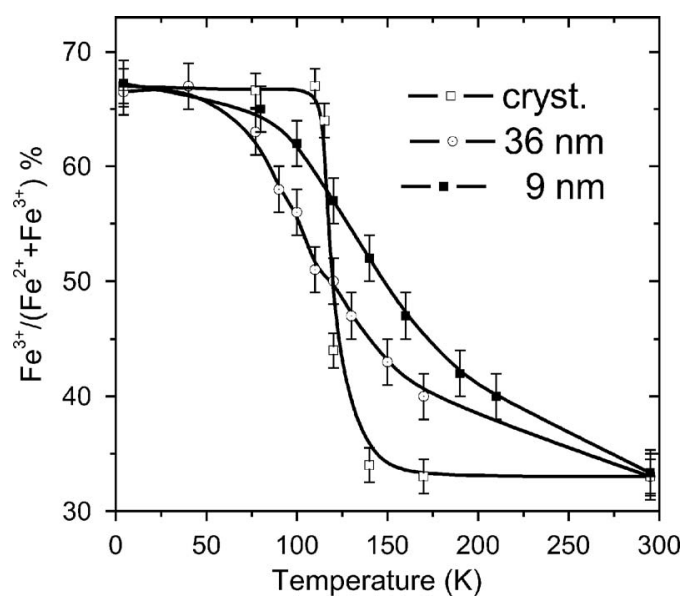

FIG. 4. The temperature dependence of the relative content ratio of $\mathrm{Fe}^{3+}$ in the magnetite samples.

are definitely in cubic phase. By cooling down the nanosized samples, spectral change began showing hyperfine interaction parameters similar to those appearing below $T_{V}$ in the bulk sample. This change suggests that fractions of the nanosized particles have higher phase transition temperatures than the bulk crystals. It means that the cubic $\rightarrow$ monoclinic phase transition occurs at higher temperature. What is the explanation for this effect? In Ref. 7, it was shown that particle aggregation resulted in magnetic composites. Therefore, the neighboring particles behave in a different way as compared to separated clusters. Indeed, the Mössbauer spectrum of the magnetic dendritelike particle was very similar to our $9 \mathrm{~nm}$ sized sample at room temperature, and transition temperatures were considerably different above $T_{V}$. Nevertheless, our fit resulted in those components appearing in the spectra of bulk magnetite. These results show that dendritelike self-assemblies can form already during precipitation in aqueous media. The phase transition of the dendrites takes place in a broad temperature range. The magnetic interaction of the particles and the different specific surface may also shift the temperature of the cubic-monoclinic transition and, finally, the phase transition takes place in broad temperature ranges.

\section{CONCLUSIONS}

The Mössbauer spectra of 9 and $36 \mathrm{~nm}$ sized nanomagnetite and bulk magnetite samples were measured in the temperature range between 4.2 and $300 \mathrm{~K}$. For bulk magnetite, the Verwey transition temperature and the temperature dependence of the hyperfine split parameters were the same as observed earlier. Similar hyperfine interaction parameters were found for 9 and $36 \mathrm{~nm}$ sized nanomagnetite samples. We observed first that the temperature dependence of the intensities of spectral components indicated a continuous phase transition taking place in a very broad temperature range. The continuous transition can be explained by the formation of fractal-like granular assemblies formed during the preparation of the samples. The hyperfine interaction values measured in these granules are close to the values measured in bulk crystals. Nevertheless, the interaction of the assemblies influences the cubic-monoclinic transition tem- 
perature. Formally, the monoclinic-cubic phase transition temperature increases for smaller particles. The selforganization of magnetic nanostructures is an interesting subject and may find potential applications in modern materials science.

\section{ACKNOWLEDGMENTS}

The work was supported by Grant No. T 32096 (OTKA).

${ }^{1}$ E. J. W. Verwey, Nature (London) 144, 327 (1939).

${ }^{2}$ E. J. W. Verwey, P. W. Haaymann, and F. C. Romejn, J. Chem. Phys. 15 181 (1947).

${ }^{3}$ M. Iizumi, T. F. Koetzle, G. Shirane, S. Chikazumi, M. Matsui, and S. Todo, Acta Crystallogr., Sect. B: Struct. Crystallogr. Cryst. Chem. B38, 2121 (1982).

${ }^{4}$ N. F. Mott, Philos. Mag. B 42, 327 (1980).

${ }^{5}$ P. Piekarz, K. Parlinski, and A. M. Oles, Phys. Rev. Lett. 97, 156402 (2006).

${ }^{6}$ S. Sun and T. C. B. Murray, J. Appl. Phys. 85, 4325 (1999).

${ }^{7}$ D. G. Mitchell, J. Magn. Reson Imaging 7, 1 (1997).

${ }^{8}$ B. J. Bonnemain, J. Drug Target. 6, 167 (1998).

${ }^{9}$ Y. Okuhata, Adv. Drug Delivery Rev. 37, 121 (1999).

${ }^{10}$ B. J. Evans and S. J. Hafner, J. Appl. Phys. 40, 1411 (1969).

${ }^{11}$ W. Kündig and R. S. Hargrove, Solid State Commun. 7, 223 (1969).

${ }^{12}$ R. S. Hargrove and W. Kündig, Solid State Commun. 8, 303 (1970).

${ }^{13} \mathrm{H}$. Topsøe and S. Morup, in Proceedings of the International Conference on Mössbauer Spestroscopy, edited by A. Z. Hrynkievicz and J. A. Sawiczki, Cracow, 1975 (unpublished), p. 321.

${ }^{14}$ G. F. Goya, T. S. Berquo, F. C. Fonseca, and M. P. Morales, J. Appl. Phys. 94, 3520 (2003).
${ }^{15}$ Z. F. Krupyanskii and I. P. F. Suzdalev, J. Phys. (Paris), Colloq. 35, 407 (1974).

${ }^{16}$ A. J. Barker, B. Cage, S. Russek, and C. R. Stoldt, J. Appl. Phys. 98, 063528 (2005).

${ }^{17}$ S. Belakrishnan, Y. K. Gun'ko, S. Tatiana, T. S. Prova, R. A. Moore, M. Venkatesan, P. Douvalis, and P. Brouke, Small 2, 819 (2006).

${ }^{18}$ J. M. J. Honig, J. Alloys Compd. 229, 24 (1995).

${ }^{19}$ T. Sugimoto and E. J. Matijevic, J. Colloid Interface Sci. 74, 227 (1980).

${ }^{20}$ P. Berger, B. Adelman, K. J. Beckman, D. J. Campbell, A. B. Ellis, and G. C. Lisensky, J. Chem. Educ. 76, 943 (1999).

${ }^{21}$ G. Ribárik, J. Gubicza, and T. Ungár, Mater. Sci. Eng., A 387-389, 343 (2004).

${ }^{22}$ T. Ungár, J. Gubicza, G. Ribárik, and A. J. Borbély, J. Appl. Crystallogr. 34, 298 (2001)

${ }^{23}$ F. J. Berry, S. Skinner, and M. E. Thomas, J. Phys.: Condens. Matter 10, 215 (1998).

${ }^{24}$ G. A. Sawatzky and J. M. D. Coey, J. Appl. Phys. 40, 1402 (1969).

${ }^{25}$ C. M. Srivastava, S. N. Shringi, and M. V. Babu, Phys. Status Solidi A 65, 731 (1981).

${ }^{26}$ M. Rubinstein and D. W. Forester, Solid State Commun. 9, 71 (1970).

${ }^{27}$ J. L. Dormann, C. Djega-Mariadossu, and V. A. M. Barbers, Proceedings of the conference on the Application of the Mössbauer Effect, edited by V. G. Bhide, New Delhi, 1982 (unpublished), p. 196.

${ }^{28}$ J. P. Wright, J. P. Attfield, and P. G. Radaelli, Phys. Rev. B 66, 214422 (2002).

${ }^{29}$ R. Aragón, D. J. Buttrey, J. P. Shepherd, and J. M. Honig, Phys. Rev. B 31, 430 (1985).

${ }^{30}$ J. P. Shepherd, R. Aragón, J. W. Koenitzer, and J. M. Honig, Phys. Rev. B 32, 1818 (1985)

${ }^{31}$ I. V. Shvetz, G. Mariotto, K. Jordan, N. Berdunov, R. Kantor, and S. Murphy, Phys. Rev. B 70, 155406 (2004).

${ }^{32}$ P. W. Anderson, Phys. Rev. 102, 1008 (1956). 